Revue d'histoire de l'Amérique française

TAL REVUE D.HISTOIRE DE L'AMÉRIQUE FRANÇAISE

\title{
La construction du premier chemin Québec-Montréal et le problème des corvées (1706-1737)
}

\section{Roland Sanfaçon}

Volume 12, numéro 1, juin 1958

URI : https://id.erudit.org/iderudit/301881ar

DOI : https://doi.org/10.7202/301881ar

Aller au sommaire du numéro

\section{Éditeur(s)}

Institut d'histoire de l'Amérique française

\section{ISSN}

0035-2357 (imprimé)

1492-1383 (numérique)

Découvrir la revue

\section{Citer cet article}

Sanfaçon, R. (1958). La construction du premier chemin Québec-Montréal et le problème des corvées (1706-1737). Revue d'histoire de l'Amérique française, 12(1), 3-29. https://doi.org/10.7202/301881ar d'utilisation que vous pouvez consulter en ligne. 


\section{LA CONSTRUCTION DU PREMIER CHEMIN QUÉBEC-MONTRÉAL ET LE PROBLĖME DES CORVÉES $(1706-1737)^{1}$}

La nécessité des chemins ne s'est faite sentir qu'assez tard en Nouvelle-France. La voie maritime du Saint-Laurent reliait toutes les régions essentielles de la colonie. De plus, le premier cheval n'arriva qu'en 1647. Ce n'était là qu'un cadeau offert au gouverneur. Cependant, en 1720, alors que la population aura atteint 24,500 âmes, il y aura déjà 5,063 chevaux. En donnant 5 membres à la famille moyenne de l'époque, il apparaît que chacune pouvait avoir son cheval. Mis en possession d'un moyen de traction terrestre, les gens pouvaient songer à éviter par la construction de chemins les incertitudes des vents pour les vaisseaux et les fatigues de la rame. ${ }^{2}$

Les chemins seigneuriaux qui existaient au début du XVIII ${ }^{\circ}$ siècle pouvaient-ils répondre convenablement aux besoins ? Parmi les 37 seigneuries qui s'échelonnent de Québec à Montréal, seulement 14 ont offert des tronçons de chemins qui furent élargis et améliorés pour servir au grand chemin royal. Sauf dans les villes, il est certain qu'aucun de ces chemins n'allait d'une seigneurie à l'autre. Limités au centre de chacune, ils permettaient de n'atteindre que l'église paroissiale ou le moulin seigneurial.

L'État allait remédier à cette situation. Depuis 1687, l'office de grand-voyer existait au pays $;^{3}$ mais jusqu'à ce moment la charge s'était résumée à très peu de chose. Une ère nouvelle s'ouvrait pour la voirie le $1^{\text {er }}$ février 1706 , lorsqu'un règlement du Conseil Supérieur de la Nouvelle-France obligea le grand-voyer

1 Condensé d'une thèse présentée à l'Institut d'Histoire de Laval en vue de l'obtention de la licence ès lettres (histoire).

2 R.-L. Séguin, Le cheval et ses implications historiques, dans RHAF, sept. 1951: 227s.

3 P.-G. Roy, Les grands-voyers de la Nouvelle-France et leurs successeurs, dans Les Cahiers des Dix, 8: 182. 
de voir à la construction des grands chemins. ${ }^{4}$ Cette date est à l'origine de la construction du premier chemin royal de Québec à Montréal. Elle fixe ainsi clairement dans le temps le début de notre étude.

\section{Première Partie}

\section{L'ENTREPRISE ET SES TECHNIQUES}

Le premier chemin Québec-Montréal, sur la rive nord du fleuve, devait avoir 175 milles de long. De Québec vers le sudouest, s'étend une vaste plate-forme où le fleuve et ses affluents coulent dans des rainures étroites, d'environ 300 pieds de profondeur à Québec, 50 pieds aux Trois-Rivières. Pour traverser les rivières ou rejoindre des habitations sur les grèves, il fallait parfois descendre brusquement de cette plate-forme jusqu'au niveau des eaux. En amont des Trois-Rivières, le lac SaintPierre fait place à la rainure et les rives sont sujettes aux inondations tous les printemps. Puis suit la plaine de Montréal, basse et uniforme. Sur ces terrains, nous avons relevé dans une vingtaine de seigneuries la présence certaine de «bois debout», qu'il a fallu abattre pour la construction des chemins. L'hiver, la neige recouvrait entièrement les chemins d'été. Les glaces et les crues printanières endommageaient les ponts, et des sols glaiseux souvent trop humides se transformaient en bourbiers.

Il y avait donc des difficultés; mais aucune d'entre elles n'était insurmontable. L'étude des techniques nous en convaincra. Quatre parties la composent: le tracé, la qualité des chemins d'été, les moyens de franchir les rivières et la qualité des chemins d'hiver.

A cette époque, la plupart des paysans avaient leurs habitations sises le plus près possible du fleuve et de leur canot. Pour relier autant ces bâtiments que les deux villes de la colonie, le chemin devait passer le plus près possible du fleuve. C'est pourquoi dans les seigneuries de Champlain, Batiscan, ${ }^{5}$ Portneuf, Berthier, d'Orvilliers, Dautré et Lanoraie, le grand-voyer Bois-

4 Jugements du Conseil Supérieur de Québec (1889), V: 238.

5 Archives de la Province de Québec, Procès-verbaux des grands-voyers, 5: 38r. Nous référerons à ces documents par le sigle GV. 
cler ${ }^{6}$ dut vers les 1732 déplacer un chemin qui allait être englouti. Quant aux seigneuries de Sainte-Anne, Batiscan et Champlain, elles ont connu jusqu'à quatre variantes dans leur tracé. ${ }^{7}$

Pour passer de Québec à Neuville, il y a deux chemins. Le voyageur Franquet nous en parle ainsi: "Le premier [par le Cap-Rouge] règne le long du fleuve et oblige à monter et à descendre plusieurs côtes, en quoy il est dûr et difficile aux chevaux; pour les éviter, on suivit l'autre [par l'Ancienne-Lorette] $\gg .8$

Une conclusion s'impose donc ! Si la localisation des habitations a trop rapproché du fleuve le tracé du premier chemin royal, on devra bientôt l'en éloigner et tendre à l'identifier au tracé actuel. Là où deux chemins se faisaient concurrence, on a vite reconnu la valeur de celui que nous préférons toujours. Si les tracés d'hier et d'aujourd'hui n'ont que peu différé, il n'en va pas de même de la qualité des chemins.

Quelle largeur avaient-ils ? En 1706 et 1707, des ordonnances demandaient pour la sortie des villes de Québec et de Montréal des chemins de 36 pieds et même de 40 pieds de large. ${ }^{9}$ Ailleurs, le grand-voyer Bécancour se contentera souvent de 18 pieds, ${ }^{10}$ même de 12 pieds. ${ }^{11}$ Ayant l'avantage de prendre un travail déjà assez avancé, Boiscler s'appliquera toujours à obtenir la largeur de 24 pieds, d'accord avec le grand règlement de voirie du $1^{\text {er }}$ février $1706 .{ }^{12}$

Ces chemins avaient-ils la rectitude souhaitable ? Bécancour avoue, par exemple, qu'il est «obligé de détourner tout court le chemin par la maligneté du Ruisseau $\gg{ }^{13}$ Les habitants se

${ }^{6}$ Le grand-voyer Boiscler, contrairement à ce qu'on fait souvent, n'ajoute jamais de « $c$ » à la fin de son nom. Voir sa signature dans GV, 5: $2 \mathrm{r}$.

7 Pour la seigneurie de Batiscan, voir aux Archives de la Province de Québec, les Ordonnances des Intendants, 5: 30v. (nous employerons le sigle OI), et GV, $1: 53$ r., $5: 25 \mathrm{v}$.

8 Franquet, Voyages et mémoires sur le Canada (Québec, 1889), 131.

9 OI, 1: 59v. et Edits, Ordonnances ..., III: 418.

10 En 1723, GV, 1: 51v. 440 .

11 En 1715, GV, 1: 38r. Bégon, en 1722. Edits, ordonnances..., III:

12 Jugements du Conseil supérieur de Québec, V : 238.

$13 \mathrm{GV}, 1$ : 22v. et suiv. 
plaindront parfois des courbes en des termes qui laissent voir qu'on aurait pu faire mieux. ${ }^{14}$ Boiscler corrigera plusieurs de ces détours. ${ }^{15}$

Cependant, même sous sa direction, le revêtement ne s'améliora guère. En forêt, on abattait les arbres à la hache, on enlevait les roches, les souches et tout autre bois. Puis, il suffisait, comme ailleurs, de labourer le sol «à hullot», c'est-à-dire dans la même direction que le chemin, et de herser. ${ }^{16}$ En aucun texte, nous n'avons rencontré qu'on obligeât les gens à recouvrir les chemins de gravier ou d'autres matériaux. Dans des endroits particulièrement exposés à se transformer en bourbiers, on recourra à un pavage de pieux de bois. Mais il ne semble pas qu'on ait réalisé souvent des travaux aussi considérables. On n'aurait sans doute pas manqué d'en parler: or seulement deux textes en font mention. ${ }^{17}$ D'une façon générale, on ne combattra pas l'humidité excessive par l'amélioration des pavés, mais par d'autres moyens.

Le plus commun d'entre eux fut le fossé. Faute d'en avoir aménagé, des bourbiers rendaient presque tous les chemins impraticables. Boiscler en fera aménager à peu près partout. Leurs dimensions minima seront de deux pieds et demi de creux et de large. ${ }^{18}$ Parfois, il faut creuser jusqu'à ce que les eaux s'écoulent. ${ }^{19}$ Ces fossés seront à « recaler » tous les ans le 15 juin. Le capitaine de milice devra rappeler aux habitants leurs obligations en ce jour. ${ }^{20}$

Souvent, on pratiquait des saignées, ou rigoles, à travers les champs. Comme, la plupart du temps, ces saignées coupaient le chemin pour permettre à l'eau de circuler à sa guise selon les pentes du terrain, des ponceaux permettaient de les franchir sans inconvénient. On leur donne régulièrement le nom de saignéepontée.

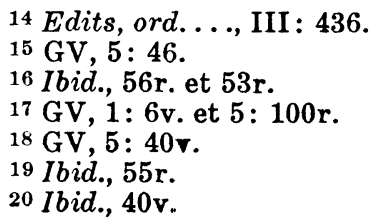


Pourtant, surtout sur les rives basses du fleuve, ces travaux ne suffisaient pas. Il fallait charroyer de la terre des régions environnantes pour former des levées. Elles offraient à leur sommet un chemin de 18 pieds de large. On les protégera contre l'œuvre destructrice des eaux en creusant de bons fossés de chaque côté. Certaines de ces levées seront assez longues pour qu'on y pratique plusieurs saignées-pontées. Elles auraient pu, autrement, former digue et immobiliser l'eau d'un seul côté. ${ }^{21}$ Ces sortes de travaux furent nécessaires très tôt; nous en avons un exemple dès $1710 .^{22}$

La terre ou le roc à remuer pour adoucir les montées ou descentes trop à pic demandait cependant un effort plus considérable encore. Ces travaux furent plus rares. La descente de la rivière Jacques-Cartier fut abaissée de dix pieds à son sommet. ${ }^{23}$ C'est là un cas extraordinaire. Les côtes resteront un grave inconvénient des chemins du régime français, un sujet de plaintes pour tous ceux qui s'en serviront. Nous en avons relevées continuellement dans les ordonnances et les procès-verbaux. Qu'il nous suffise de reproduire ici le témoignage du voyageur Franquet. En allant de Montréal à Québec par terre en mars 1753, il fut, raconte-t-il, «fatigués de mille façons, soit à monter ou à descendre les côtes, et toujours à la veille de s'y précipiter du haut en bas . $^{24}$

Sur les grèves, enfin, les charrois s'enlisaient assez facilement dans les sables. Pour cette raison, Boiscler devra changer le tracé du chemin dans la seigneurie de Champlain.25 A l'embouchure de la rivière Jacques-Cartier, il fallut planter des balises dans le sable pour l'empêcher de se déplacer continuellement et d'occasionner avec lui des changements fréquents dans le tracé du chemin. ${ }^{26}$

La largeur officielle du chemin du roi était donc de 24 pieds; mais on tolérait, surtout au début, qu'il fût plus étroit. On en

21 Ibid., 2v.

$22 \mathrm{GV}, 1$ : 35v., $54 \mathrm{v}$.

$23 \mathrm{GV}, 2: 49 \mathrm{v}$.

24 Voyages ...., 163.

$25 \mathrm{GV}, 5$ : $38 \mathrm{r}$.

$26 \mathrm{GV}, 2$ : 49v., 5 : 15v. 
exigeait de plus large à la sortie des villes. On s'efforce de le rendre le plus droit et le plus praticable possible; mais il reste assez sinueux et n'a ordinairement aucun pavé spécial. On se contente d'un simple hersage. Les fossés, les saignées-pontées, les levées et quelques creusements de terrain au sommet des côtes demeurent les travaux les plus avancés. Autant que possible, on évite les sables. Tel est l'aspect de ce premier chemin de Québec à Montréal.

Mais il fallait aussi franchir les nombreux affluents du Saint-Laurent. Trois possibilités se présentaient: les gués, les ponts et les véhicules flottants. Selon les dimensions des cours d'eau, les trois seront en usage en Nouvelle-France.

En 1723, Bécancour parle d'un chemin dans la seigneurie de Portneuf, qui « descendra Le Long de La Rivière [on n'en donne pas le nom] pour aller trouver Le guay ordinaire Laquelle Rivière passés ... ${ }^{27}$ Il est évident qu'on ne fait pas de pont à cet endroit. Lorsque Boiscler commencera ses visites des chemins en 1731, la plupart des ponts sur les fossés et les ruisseaux ne seront pas faits. ${ }^{28}$ Sous son ordre, on en construira partout, semble-t-il.

Tous les ponts de la Nouvelle-France étaient faits de bois, car on en trouvait toujours à la portée de la main. Le grandvoyer pouvait même exiger le cèdre, qui a toujours montré une meilleure résistance à l'eau. Que valaient ces ponts ? Dès l'arrivée de Boiscler en $1731,{ }^{29}$ ils auront une qualité qui ne variera à peu près pas jusqu'à l'achèvement du chemin. On prépare de chaque côté de la rivière de bons chevalets au sommet desquels on place une grosse poutre de bois. Cinq lambourdes de cèdre, fixées sur ces poutres de trois pieds en trois pieds, réunissent les deux rives. Leur longueur et leur grosseur varient avec l'importance du cours d'eau (on ne donne jamais plus de détails sur ces lambourdes). On couvre le tout de pieux écarris, de

$27 \mathrm{GV}, 1: 35 \mathrm{v}$.

$28 \mathrm{GV}, 2: 3 \mathrm{r}$.

29 Auparavant, Bécancour reste vague. On lit souvent dans ses procèsverbaux: * à lesgard des ponts, ils seront faits de cèdre propre à soutenir le poids des charois $\gg$. GV, 1: 12r. 


\section{LE PONT MODÈLE DU RÉGIME FRANÇAIS}

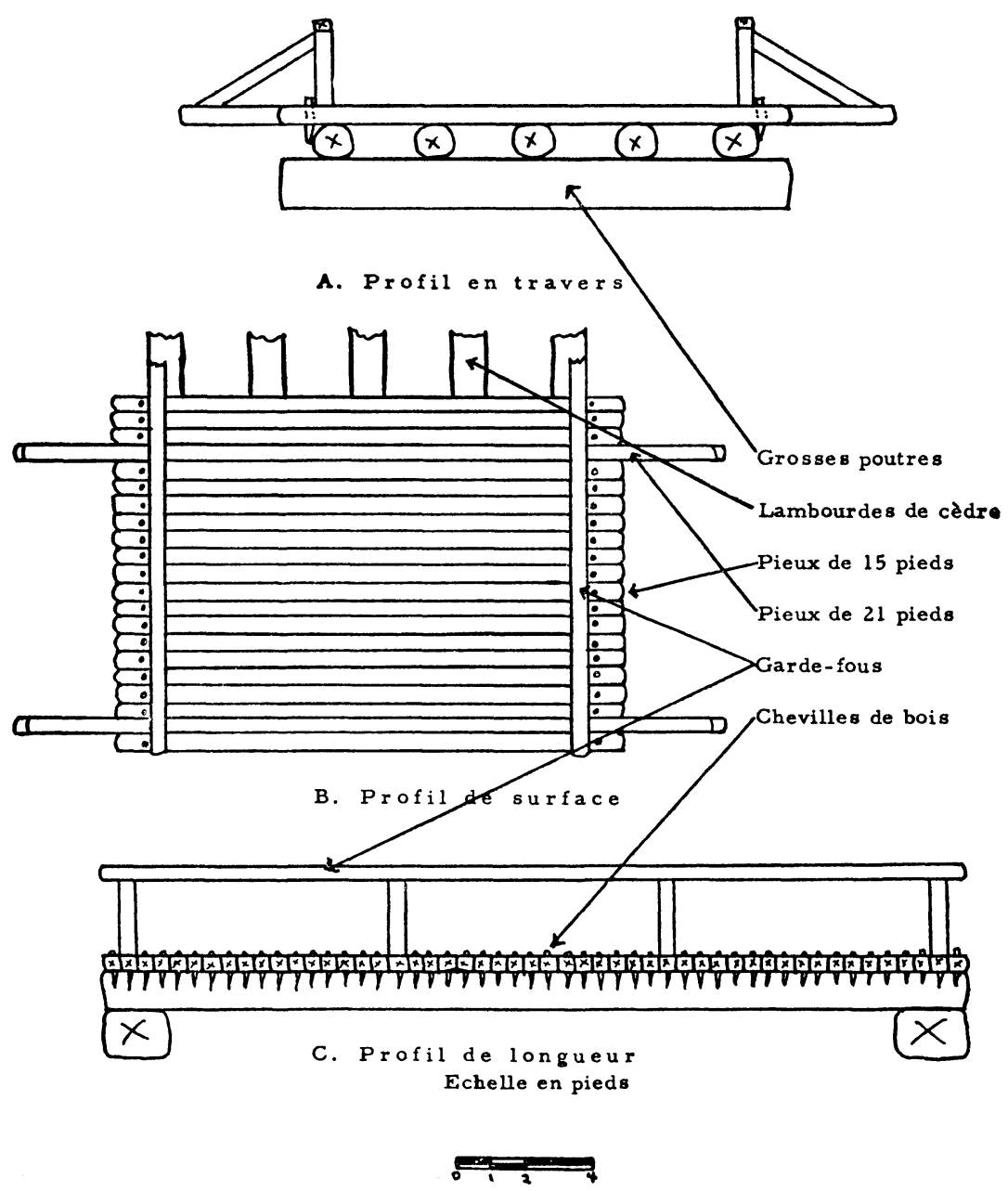


quinze pieds de long et de six pouces d'épais. Ces pieux sont retenus sur les lambourdes au moyen de chevilles de bois plantées à leurs extrémités. De neuf pieds en neuf pieds (plus tard, ce sera huit), on intercalait des pieux de 21 pieds de long qui excédaient de trois pieds de chaque côté du pont. Sur ces rallonges, on pouvait appuyer les poteaux qui soutenaient un garde-fou. ${ }^{30}$

Tel est le pont modèle du temps. Mais une donnée nous manque: la longueur des lambourdes. Il est donc assez difficile de connaître la largeur maxima des rivières que l'on pouvait ponter. Le cas de la rivière Mascouche vient nous éclairer. Elle avait 80 pieds de large. En 1733, on décide d'y faire un pont sans pilier. Grâce à des constructions de pierre et de bois qu'on aménagera de chaque côté de la rivière, la distance à couvrir par les lambourdes ne sera plus que de 38 pieds. ${ }^{31}$ Ce devait être la longueur maxima de toute lambourde. A-t-on souvent fait usage de piliers au centre des rivières pour soutenir les ponts? C'est la seule mention que nous en ayons trouvée et ce n'est que pour s'en interdire la construction. D'autre part, la rivière Mascouche nous donne le seul exemple de travaux aussi considérables (du moins sur notre chemin royal, jusqu'en 1737). Même si nous acceptons la possibilité de quelques petits aménagements spéciaux en bien des cas, nous ne pouvons nous empêcher de croire que la longueur maxima des ponts normaux ne devait guère dépasser 40 pieds. Partout ailleurs, on recourait au canot ou au bac.

En 1710 , on mentionne la présence d'un canot sur un affluent de la rivière Sainte-Anne. ${ }^{32}$ A partir de 1731, les bacs remplaceront les canots. ${ }^{33}$ Ces bacs avaient des conducteurs spéciaux nommés passeurs, qui recevaient leur titre de l'intendant et dont les émoluments, perçus sur les passagers, étaient

30 On peut consulter pour l'ensemble de ces détails les documents suivants: GV, $2: 1 \mathrm{v}$. et $34 \mathrm{v}$., $5: 27 \mathrm{r}$.

$31 \mathrm{GV}, 5: 102 v$. et suiv.

$32 \mathrm{GV}, 1: 11 \mathrm{r}$.

$33 \mathrm{GV}, 5$ : 43v. 
aussi entièrement fixés par l'intendant. ${ }^{34}$ Le bac est assez lourd pour qu'il soit nécessaire d'organiser une corvée générale de tous les habitants pour aider le passeur à le sortir de l'eau à l'automne et à l'y remettre au printemps..$^{35}$ Il ne peut naviguer que là où l'eau a une profondeur de deux pieds et demi. ${ }^{36}$ Nous n'avons malheureusement pas trouvé d'autres précisions à son sujet.

On traversait en bac les rivières suivantes: Cap-Rouge, Jacques-Cartier, Sainte-Anne, Batiscan, Champlain, Saint-Maurice, Grand-Yamachiche, Rivière-du-Loup, Maskinongé, Berthier, Grand et Petit-Chicot, des Prairies. ${ }^{37}$

Si, l'été, les rivières retardaient la circulation, l'hiver, les glaces offraient des ponts naturels partout. Cependant, l'hiver était aussi cause d'un grand nombre d'inconvénients lorsque les neiges abondantes engloutissaient le chemin d'été. On préférait alors les glaces du fleuve, surtout dans la région du lac SaintPierre. $^{38}$

Des ordonnances sont nombreuses, qui demandent aux habitants résidant «sur les grands chemins, de placer aux premières neiges des balises, de vingt-quatre pieds en vingtquatre pieds, le long du front de leur habitation, et de les entretenir, lesquelles balises seront au moins de la hauteur de six pieds ».39 Après chaque «bordée » de neige, il faudra aussi faire aller et venir les bestiaux entre ces balises. ${ }^{40}$ Ces ordonnances étaient-elles bien exécutées ? On peut en douter, car le gouverneur prend soin de se faire précéder par le grand-voyer lorsqu'il se rend de Québec à Montréal en hiver. ${ }^{41}$ Encore ici, la théorie

34 Edits, ord...., II : 366s.

35 GV, 5: 119 .

36 Ibid., $118 \mathrm{v}$.

37 On peut consulter GV, 5: 117v. et Edits, ord...., II : 367s. Dans les Voyages... de Franquet, on verra aux pages 160 et 171 qu'il traverse sur glace les rivières Jacques-Cartier et Cap-Rouge, alors que pour d'autres on se sert de ponts.

38 Edits, ord...., III : 428.

39 Ibid., 457.

$40 \mathrm{OI}, 12 \mathrm{~A}: 12 \mathrm{r}$.

41 En ce cas, le grand-voyer est payé pour chaque heure de travail, car cela ne lui incombe pas normalement de par son office. Franquet, Voyages, 145. 
demandée est différente des réalités obtenues. A la base de toute cette entreprise, il y a les hommes.

\section{Deuxième Partie}

\section{L'ENTREPRISE ET LES HOMMES}

L'étude du phénomène humain dans la construction du premier chemin Québec-Montréal comprendra d'abord un exposé des institutions de la voirie. Nous en analyserons ensuite le fonctionnement dans le concret.

Les institutions de la voirie s'encadrent dans l'ensemble des institutions de la Nouvelle-France. Nous savons que l'intendant était le chef de l'administration de la «justice, police et finance ». A ce titre, il devait voir à toute l'organisation interne du pays; de lui, dépendait donc en premier lieu la voirie. Cependant, comme il avait les nombreuses autres charges de l'administration générale, il confiait la voirie au grand-voyer. Comme à son tour, celui-ci était bien occupé à tracer les chemins à travers tout le pays, leur construction était dirigée par les capitaines et les officiers de milice de chaque paroisse ou seigneurie: ils recevaient, de ce fait, le pouvoir de commander aux habitants de leur localité. Chaque habitant se voyait forcé de fournir gratuitement le terrain et les travaux nécessaires à la construction du chemin. C'était les corvées. De l'intendant au moindre habitant, tous les gens étaient mis en mouvement pour les travaux de voirie.

La répartition des tâches n'est toutefois pas des plus rigoureuses. L'intendant doit intervenir continuellement. Ils sont nombreux les cas où le grand-voyer est parti tracer des chemins sur l'ordre exprès de l'intendant, ${ }^{42}$ qui demeure toujours libre d'approuver ou de modifier les décisions du grandvoyer. ${ }^{43}$ L'intendant donnera aussi des ordres directement aux capitaines de milice. ${ }^{44} \mathrm{Il}$ contrôle parfois de près la marche des travaux ${ }^{45}$ et inflige des amendes aux habitants qui refusent de




se conformer aux autorités. ${ }^{46}$ L'intendant n'avait donc pas qu'un rôle indirect dans la construction des chemins. Si le grandvoyer est immédiatement responsable devant lui, les habitants et les capitaines de milice ne sont guère plus éloignés de son attention. On dira que son intervention ne se fait que dans les cas difficiles; mais ils étaient tellement nombreux (voir Graphique page 21). Entre le 1er février 1706 et le 14 février 1737 (dates du début de la construction du chemin royal et de son achèvement convenable), les grands-voyers Bécancour (16891729 ) et Boiscler (1731-1750) ont rédigé 127 procès-verbaux tandis que les intendants Raudot (1705-1710), Bégon (17101725), Dupuy (1725-1728) et Hocquart (1729-1748) émettaient 33 ordonnances. Il apparaissait plus d'une ordonnance pour 4 procès-verbaux. Les intendants ont donc joué un rôle considérable dans l'ensemble de l'entreprise.

Cependant, le plus grand travail fut fait par les grandsvoyers. Ceux-ci reçoivent leur office du roi et peuvent être révoqués selon son bon plaisir. ${ }^{47}$ Le règlement de voirie du Conseil supérieur de Québec leur demande de tracer les chemins «de concert avec Les propriéttaires des Seigneuries, Les of $\mathrm{f}^{\text {ers }} \mathrm{de}$ Milice en Leur absence s'il n'y a pas de juge et Six des plus anciens et Considérables habitants du lieu ».48 Le grand-voyer visite toutes les seigneuries, examine attentivement, écoute et, à l'occasion, suit les conseils de chacun. Dans les «bois debout», des plaques à la hache sur les arbres délimitent le tracé du futur chemin; dans les champs et les jardins, on plante des piquets. Parfois, le grand-voyer doit remettre sa visite à plus tard, soit parce que les gens auxquels il doit demander conseil sont absents, ${ }^{49}$ soit que l'on préfère attendre la chute des feuilles pour mieux observer les terrains dans les bois, ${ }^{50}$ soit que l'on n'ose pas trop se promener dans les champs de blé. ${ }^{51}$ Ce sont là des petits détails; mais ils nous laissent pénétrer dans l'atmosphère

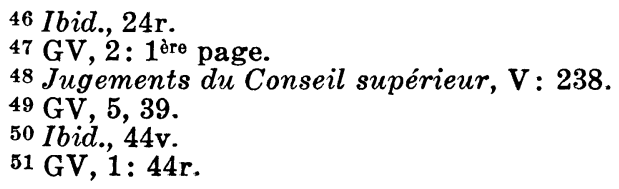


d'une époque révolue. En présence de tous ceux qui l'ont accompagné, le grand-voyer rédige ensuite un procès-verbal dans lequel on note toutes les décisions prises au sujet du tracé du chemin, des endroits à ponter et de l'organisation du travail.

Même si le grand-voyer surveille plus ou moins directement ses procès-verbaux, leur contenu est fondamentalement réalisé grâce aux capitaines de milice. Ils en font la lecture après la messe paroissiale, le dimanche, pour que personne n'ignore ce qu'il doit faire. Chaque habitant n'a plus qu'à se soumettre aux ordres et aux corvées.

Cependant, on ne connaîtra toujours qu'une partie de ces institutions, si les idées fondamentales, qui sont à la base de la répartition des corvées entre les habitants, ne nous sont pas révélées. On peut en compter trois: le principe de concession, l'idée d'utilité et l'idée de compensation.

Parce que «leurs contrats de concession les y obligent $\gg, 52$ dès 1706 l'intendant Raudot peut décréter «que chaque habitant par où le chemin passe sera tenu de faire tous les travaux nécessaires pour le rendre praticable le long de son habitation $\gg .^{53}$ Fournir gratuitement terrain et travaux sur sa concession, tel est le contenu du principe de concession. On n'y déroge pas facilement. Quelques-uns devront déplacer leurs bâtiments. ${ }^{54}$ Sur leur domaine, les seigneurs y sont soumis, qu'ils soient laïcs ${ }^{55}$ ou religieux. ${ }^{56}$ Il en va de même pour ceux qui n'habitent pas encore leur concession. ${ }^{57}$ Le cas des terres administrées par des fermiers ou des tuteurs semblait assez complexe. Dans ces deux cas, les véritables propriétaires devront rembourser la valeur du travail de leurs substituts. ${ }^{58}$

On tenait ferme à ce principe parce qu'on pouvait l'appliquer facilement. En effet, si nous excluons les domaines des seigneurs, les dimensions des terres de la Nouvelle-France étaient assez

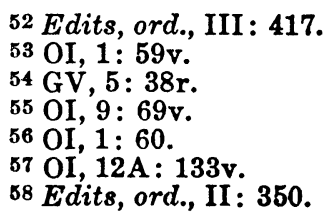


égales: environ 2 à 3 arpents de largeur par 40 à 60 arpents de profondeur. Ces terres étaient, de plus, toutes orientées perpendiculairement au fleuve et au chemin, qui avaient la même direction. La longueur moyenne de chemin que chacun devait fournir était donc de 2 à 3 arpents. Cette circonstance faisait du principe de concession une règle apte à donner à chacun une part relativement modique et assez égale des charges. Nous comprenons mieux ainsi pourquoi on ne faisait pas normalement le travail en commun.

Mais le travail en commun n'est pas exclu. Dans des cas particuliers, on pourra faire le partage des tâches selon l'idée d'utilité (on ne saurait parler d'un principe, car elle n'en a pas la force). Cette idée permet aux autorités (grand-voyer ou intendant) d'exiger des gens des corvées supplémentaires dans la mesure où ceux-ci tirent ou tireront des avantages de certaines difficultés à surmonter. Ces difficultés peuvent être de deux catégories complètement distinctes.

C'est d'abord le travail qui, une fois terminé, donnera des effets très utiles à ceux qui l'auront fourni. Ainsi, la construction d'un chemin sera partagée entre les habitants, «chacun à proportion de l'usage du dit chemin ${ }^{59}$ Cependant, il semble qu'on recourt à cette idée le moins souvent possible. Une longue dispute nous le prouve. Des concessionnaires déjà chargés de corvées voulaient être exemptés de travaux dont les véritables bénéficiaires allaient être les habitants de la localité suivante. Parce que c'était un chemin royal, le principe de concession prévalait. Les concessionnaires durent construire le chemin. Les bénéficiaires ne furent chargés que de son entretien. ${ }^{60}$

L'idée d'utilité est aussi présente lorsque les difficultés à surmonter ont été créées pour l'utilité qu'on en tire en dehors des préoccupations de voirie. Ainsi en 1710, Bécancour décrète que « a l'égard des fosses qui s'y rencontrent, les ponts seront construits par ceux qui les ont faict faires pour Leurs utilités $\rrbracket^{01}$

59 Edits, ord., III : 417.

60 OI, $6: 24$ r.-25r.

$61 \mathrm{GV}, 1: 14 \mathrm{r}$. 
Si un fossé sert à deux habitants, Boiscler verra à ce que tous deux contribuent à le ponter, et ainsi de suite. ${ }^{62}$

C'est un souci de justice qui a fait naître l'idée d'utilité. C'est pour la même raison qu'est apparue l'idée de compensation. Elle permettait aux autorités d'organiser des corvées supplémentaires, lorsque des difficultés particulières se présentaient pour un habitant et l'auraient, de par le seul principe de concession, obligé à une somme de travail plus grande que celle que devait fournir le concessionnaire moyen. L'excédent de travail était alors compensé par une aide qui lui était proportionnelle.

De tels cas étaient assez fréquents. Lorsqu'au hasard des tournants, le chemin venait à changer complètement de direction et à monter sur les terres en profondeur au lieu de les traverser en largeur, c'eût été une charge trop lourde pour la donner à un seul concessionnaire: les terres étant toutes, comme nous le savons, assez étroites, mais très profondes. Le chemin suivait alors généralement les lignes de partage, pour que chacun des deux voisins pût fournir une seule moitié du terrain nécessaire. ${ }^{63}$ Souvent, lors de corvées générales, on obligeait tous les gens à construire le chemin ${ }^{64}$ ou seulement à creuser les fossés. ${ }^{65}$ Parfois, il n'y avait aucune aide, mais les deux voisins étaient dispensés des autres corvées générales. ${ }^{66}$

Le partage du travail des ponts n'est pas plus simple. En $1708^{67}$ comme en 1732 , on veut que «Les ponts qui sont sur les Rivière ou sur Ruisseau naturel [...] [soient] faits par tous Les habitans ${ }^{68}$ Comme nous l'avons vu, l'origine artificielle des cours d'eau constituait un argument assez décisif pour en donner le pontage à ceux qui en tiraient de l'utilité; mais il semble que, d'une manière générale, on considère moins leur origine naturelle que la seule idée de compensation lorsqu'on décide d'organiser des corvées supplémentaires pour la cons-

$62 \mathrm{GV}, 5: 27 \mathrm{r} ., 1: 22 \mathrm{v}$.

$63 \mathrm{GV}, 5: 52$ r., Edits, ord., III : 442.

$64 \mathrm{GV}, 1: 73 \mathrm{v}$.

$65 \mathrm{GV}, 5: 57 \mathrm{r}$.

$66 \mathrm{GV}, 1: 38 \mathrm{r}$.

67 Ibid., $4 \mathrm{r}$.

$68 \mathrm{GV}, 5: 38 \mathrm{v}$. 
truction des ponts. En n'ayant présent à l'esprit que cette seule idée, le grand-voyer pourra juger si le propriétaire seul doit tout faire, ${ }^{69}$ ou si un, ${ }^{70}$ deux, ${ }^{71}$ six, $^{72}$ vingt-sept ${ }^{73}$ ou tous les habitants ${ }^{74}$ doivent l'aider et compenser pour l'insuffisance de ses seuls moyens. C'est réellement là la façon de procéder la plus fréquente. Quant à l'entretien de ces ponts, il est tantôt laissé au seul propriétaire, même si la construction a été faite par corvée générale ;5 tantôt il retombe, comme sa construction, sur tous les habitants. ${ }^{76}$ Ce deuxième cas est toutefois beaucoup moins fréquent.

L'aménagement des côtes difficiles fait aussi apparaître l'idée de compensation. Boiscler n'invoquera pas d'autre raison: "Comme la ditte Coste [écrit-il] est très difficile a faire a cause des Buttes quil faut oster et abbatre et seignier pontés quil faut faire, elle sera faite et Entretenüe par tous Les habitans. ${ }^{77}$ Nous voyons donc aussi que son entretien peut retomber sur tous les habitants; mais il arrive aussi qu'on le laisse à quelques voisins de la côte, ${ }^{78}$ ou à son propriétaire seul «a moins quelle ne fut Beaucoup endommagée auquel cas elle sera racommodée par Corvée . $^{79}$

Il serait sans doute superflu de recommencer la même étude en détail sur la construction de plusieurs levées et le creusement de fossés particulièrement difficiles; qu'il suffise de dire qu'on emploie toujours le même système complexe et imprécis. ${ }^{80}$ Cependant, deux cas, où l'idée de compensation s'applique d'une manière spéciale, méritent une plus grande attention; cela se




produit sur les grandes rivières qui n'appartiennent qu'à l'Etat, et encore sur les terres non concédées.

Qui donc devra faire les bacs ou canots sur les rivières ? En 1732, Boiscler décrète qu'un « Canot et Cordes seront fait et fourny par Le Public $\gg{ }^{81}$ En parlant des habitants de Lachenaie, il remarque «que Les dits habitans n'ont pas mis une Corde au Bacq de la Rivière Mascouc[h]e Comme nous L'avions ordonné ${ }^{82}$ Si tous doivent fournir la corde, il semble assez sûr de conclure qu'il en va aussi de même pour le bac. Dans les deux cas, il y a donc corvée générale. Le chemin et les descentes qui conduisent au bac sont aussi faits et entretenus par tous les habitants. ${ }^{83}$ Mais, comme le concessionnaire riverain fournit le terrain, c'est à lui qu'on remet les titres et l'exclusivité de passeur. ${ }^{84}$

Sur les terres non concédées, une coutume s'est imposée par la force des choses : la corvée générale. ${ }^{85}$

Partout où l'idée de compensation entre en jeu, il peut donc y avoir corvée générale. Mais jusqu'où s'étendait géographiquement l'obligation pour les habitants ? La seigneurie sert de cadre normal. ${ }^{86}$ Si elle est trop grande ou trop peuplée, on fixe d'autres limites souvent paroissiales. ${ }^{87}$. Si elle est trop petite, elle peut se trouver incluse dans les limites paroissiales plus grandes. ${ }^{88}$ Le travail était ensuite partagé entre «tous les habitans de la ditte seigneurie au prorata des terres qu'ils y possedent $\gg^{89}$ y compris les seigneurs. ${ }^{90}$ C'était le capitaine de milice, souvent

$81 \mathrm{GV}, 5: 35 \mathrm{v}$.

82 Ibid., 43v.

83 Ibid., 115v., $117 \mathrm{r}$.

84 Ibid., 166r.

85 OI, 13: 13v.

$86 \mathrm{GV}, 5: 4 \mathrm{v}$.

87 OI, 19 : 33r. Edits, ord., III : 417.

88 Ainsi la seigneurie de Dautré dans les paroisses de Lanoraie et Lavaltrie, OI, $12 \mathrm{~A}$ : $134 \mathrm{r}$.

89 Cependant, cette coutume de partager au prorata des terres ne se trouve clairement exposée qu'à partir des environs de 1732. Parfois, on a réparti les tâches également entre tous les habitants (OI, 19: 33r.), une fois en tirant au sort (en 1706, OI, 1: 31r.).

90 GV, 1:24v. 
aidé par le curé ${ }^{91}$ ou quelques habitants, ${ }^{92}$ qui déterminait concrètement à chacun la part des travaux qui lui revenait. ${ }^{93}$

Que pouvons-nous conclure de notre petit voyage à travers l'organisation des travaux de voirie ? D'une part, on ne prend jamais en considération la personnalité des gens. On ne fait aucune distinction entre le seigneur et ses censitaires. Contrairement à la coutume en vigueur en France, les ressources « en bras, en charrettes et en chevaux ${ }^{94}$ n'entrent pas en ligne de compte: l'orphelin incapable doit payer son tuteur, le concessionnaire qui n'habite pas encore sa terre, doit faire sa part entière. Si on vient en aide à quelqu'un, ce n'est pas parce que cet individu en particulier ne pourrait faire son travail, mais parce que les obstacles qui se rencontrent sur sa concession obligent l'organisation de corvées supplémentaires.

D'autre part, on s'efforce de tout concentrer sur la propriété terrienne. Le principe de concession est si important qu'en réalité, les idées d'utilité et de compensation ne sont que des exceptions à cette grande règle. Sur les chemins en profondeur, dans la construction des ponts, l'aménagement des côtes, des levées et des pavés, ou le creusement des fossés, n'avons-nous pas vu comment d'une fois à l'autre les autorités s'efforcent d'en laisser le plus possible aux concessionnaires, pour ne pas affaiblir le principe de concession. Cette conclusion s'imposera d'autant plus clairement si nous constatons que seulement, lorsqu'il n'y a pas de concessionnaires (canots ou bacs sur grandes rivières, chemin sur terres non concédées), une règle s'applique sans exception: la corvée générale. C'est qu'il n'y a rien à craindre pour le principe de concession!

N'est-on pas aussi en voie de créer une nouvelle coutume canadienne ? L'emploi des corvées générales pour les chemins en profondeur semble devenir une règle; mais le grand-voyer se contente parfois d'aider les concessionnaires autrement. On énonce bien que les ponts sur les cours d'eau naturels doivent être

91 OI, $1: 31 \mathrm{r}$.

$92 \mathrm{GV}, 5: 58 \mathrm{v}$.

93 Ibid., 30v.

94 Fr. Olivier-Martin. Histoire du droit français. Paris, 1951. 590. 
construits par corvée générale; mais souvent le concessionnaire doit tout faire ou un nombre restreint de personnes viennent l'aider. Une côte importante est normalement aménagée par tous les gens ; mais le grand-voyer peut réduire l'aide surtout pour son entretien. Tout est encore plus imprécis dans le cas de certains fossés, pavés ou levées. La corvée générale elle-même a, comme règle de base, les cadres géographiques de la seigneurie; mais le grand-voyer détermine souvent d'autres limites. Une règle, cette fois assez déterminante, exige le partage des corvées générales «au prorata des terres»; mais on a fait quelques fois une répartition égale des tâches. Il est donc évident qu'on ne procède pas sans aucune base: partout il y a une formule plus souvent employée, qui tend à devenir une coutume; mais qui n'a pas encore atteint ce stage.

Telles sont donc les institutions de la voirie! Elles ont sans doute évolué au cours de la construction du chemin. Mais cette seule évolution ne saurait expliquer l'écart qui existe entre la lenteur des réalisations de Bécancour et la rapidité de celles de Boiscler, entre l'à peu près des travaux du premier et la perfection (qui reste relative) de ceux du second.

Un simple coup d'œil sur le Graphique nous fait mieux comprendre. L'intervention de l'État fut trop peu sérieuse avant 1730. Ceci est assez étonnant car en 1710 Bécancour avait rédigé 12 procès-verbaux. Il n'en fera jamais plus de 4 par année pendant tout le reste de sa carrière (nous ne comptons que ceux qui servent à l'avancement de notre chemin). Les intendants Bégon et Dupuy ne furent pas plus vigoureux.

Non seulement, cette intervention est irrégulière, mais les textes nous montrent un Bécancour faible devant les habitants, souvent réduit à se soumettre à leur volonté. ${ }^{95} \mathrm{Si}$ ceux-ci ne s'entendent pas dans leurs demandes, on se perd en discussions in-

$95 \mathrm{GV}, 1: 68 \mathrm{r} ., 53 \mathrm{r}$. 


\section{GRAPHIQUE}

FREQQUENCE DES PROCÈS-VERBAUX ET DES ORDONNANCES (d'après l'Inventaire des procès-verbaux des grands voyers de P.-G. Roy)

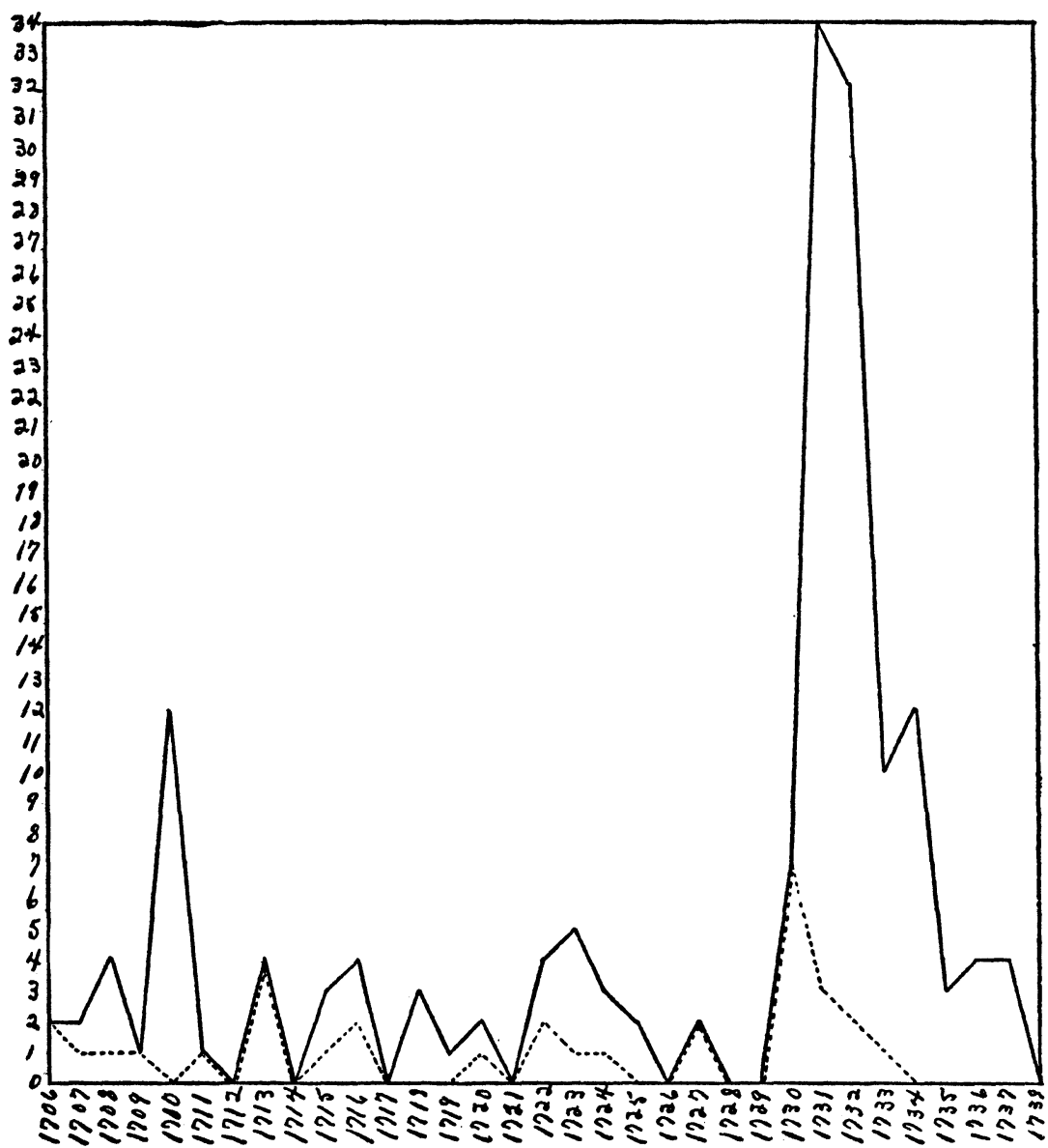

Courbe des procès-verbaux et des ordonnances

...- Courbe des ordonnances 
terminables..$^{96}$ S'ils s'entendent pour ne pas accepter le tracé proposé par les autorités, il faut tout recommencer plus tard ${ }^{97}$ ou tolérer un chemin à peu près impraticable. Cette faiblesse plus que normale des autorités va sans doute agrandir les difficultés du régime des corvées. Mais cette circonstance nous permet de les mieux observer et d'en déceler plus facilement les origines.

La plupart des gens ne voulaient pas abandonner leurs terres en culture. Voilà la première explication! En 1731, Boiscler constate que «presque tous Les habitans ne Laissaient a peine que Le Passage d'une Charette Entre Les Grains Labourant et Ensemancant Le dit Chemin ce qui forme des ornières et Bourbiers et Cause Entre Eux des difficultés parcequ'on Est Dobligation de passer dans Les grains Le dit chemin Estant par Endroits Impraticables ». ${ }^{98}$ D'autres «les [chemins] ferment et Bouchent avec des Barrières ».99

Le fond du problème semble résider dans un conflit d'idées. Les contrats de concession exigent bien qu'on cède le terrain pour la construction du chemin; mais il n'y a pas, à ce moment, un acte d'expropriation. Par le seul fait de travailler eux-mêmes à leur bout de chemin, les gens s'en croient toujours propriétaires. En ce cas, n'est-il pas normal qu'ils soient tentés d'utiliser ce terrain à leur propre profit au lieu de le donner gratuitement au public ? Découlant directement du principe de concession, ce problème ne pouvait pas disparaître.

Cependant, même lorsque les habitants n'ont pas de terres à fournir (ponts ou chemins sur terres non concédées), même lorsque les terres n'ont qu'une valeur secondaire (bois debout), on déplore continuellement des difficultés. Des buttes, des

96 Une contestation qui occasionna la publication de trois ordonnances et de deux procès-verbaux est réglée par l'intendant Bégon le 11 juin 1720 . Il ordonne \& que l'ordonnance rendüe par $M^{r}$ Raudot le 8 nov. 1711 et celle par nous rendüe le 7 de ce mois seront Exécutés Selon leur forme et teneur 》 (OI, 6: 377r.). On a donc perdu neuf ans sans obtenir aucun changement. Est-ce un cas isolé ? Loin de là ! Aux Grondines un procès-verbal de 1710 est encore en contestation en 1716 (Edits, ordon., III : 436). Souvent, on perd un an (OI, 2: 40r.) ou deux (OI, 17, 95).

97 OI, 13: 12v., GV, $1: 53 \mathrm{r}$.

$98 \mathrm{GV}, 2: 49 \mathrm{r}$.

$99 \mathrm{GV}, 5: 30 \mathrm{v}$. 
trous, des fredoches, des souches et même des arbres abattus embarrassent le chemin; ${ }^{1}$ les ponts font "Courir Risque au voyageur et à leur Chevaux de se Casser Les Jambes [...] et n'ont pas La Largeur [... ] pour passer Librement toutes sortes de voitures $\gg^{2}$ Les corvées accablaient les gens. Cette répugnance, en plus de constituer en soi un grand problème, en aggravait un autre.

Un chemin, en effet, ne peut être utile lorsqu'il est sans solution de continuité. Il serait donc préférable d'en confier la construction à un seul organisme. Or, le principe de concession partageait les travaux en autant de petites identités qu'il y avait de concessions différentes. Souvent, il n'y aura pas de chemin «par la mauvaise volonté de quelques habitants dont le Public souffre $\gg^{3}$ La répugnance générale pour les corvées multipliait volontiers le nombre des «contrevenants» et encourageait l'inaction des autres.

L'autorité était le seul facteur d'unité. Mais concrètement, l'autorité immédiate et agissante réside chez le capitaine de milice. Qu'il soit malade, ${ }^{4}$ ou qu'on se moque de ses ordres, cela suffit à «authorisé presque tous Les habitans a Labourer et Ensemancer Le Chemin de Roy $\gg .^{5}$ Nos arguments théoriques sont confirmés par les faits: nous sommes en présence d'une organisation qui nécessite une intervention continuelle de l'autorité, du capitaine ou des officiers de milice.

Or, le capitaine de milice n'était pas un fonctionnaire spécialisé dans la voirie. Il obéissait, au contraire, à tous les ordres de l'intendant ou du gouverneur. En conséquence, il ne pouvait être immédiatement responsable devant le grand-voyer et c'est parfois avec plus ou moins de zèle qu'il s'adonnait à la lourde tâche de faire exécuter les procès-verbaux. De peur de leur faire perdre leur prestige, jamais les grands-voyers ne dénonceront clairement la négligence de certains capitaines de milice,

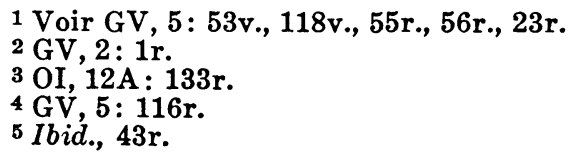


mais Boiscler plus tard nous découvrira les choses assez nettement lorsqu'il rendra les capitaines passibles d'amendes. ${ }^{6}$

Enfin, le caractère et la mentalité des habitants y seraient aussi pour quelque chose. Ne les voit-on pas tenir tête au grandvoyer, se moquer des capitaines de milice, souvent se quereller entre eux pendant plusieurs années ? Un esprit d'indépendance pourrait bien expliquer certaines contraventions ; mais la rivalité qui soulevait les habitants les uns contre les autres saurait peutêtre mieux rendre compte des longues contestations. ${ }^{7}$

Pourtant, malgré ces difficultés, le système des corvées n'était pas dépourvu de possibilités de réalisation. Avec l'arrivée de Hocquart en 1729 et de Boiscler en 1731, tout va changer. Dès l'année suivante, 7 ordonnances porteront sur le chemin qui nous occupe. En 1731, Boiscler ne fera pas moins de 34 procèsverbaux pour égaler dès sa première année tout ce qu'avait fait Bécancour en près de vingt ans. Il montrera autant d'activité l'année suivante et dès 1733 , il devra ralentir considérablement son train tant les procès-verbaux antérieurs devaient occuper suffisamment les habitants.

Ces décrets étaient exécutés. En 1734, Boiscler pourra affirmer à Hocquart «que la communication [...] [devenait] aussi libre par terre qu'elle l'a été jusques à présent par eau ». ${ }^{8}$ Comment expliquer une telle soumission des habitants au régime des corvées?

Nous avons trouvé plusieurs raisons. La plupart d'entre elles, il est vrai, valaient sous Bécancour; mais il ne sut pas leur donner l'élan réalisateur. Ainsi, ne pouvait-on pas profiter de la préparation des Canadiens au régime des corvées ? Depuis leur arrivée en Amérique, ils en avaient conservé le principe par les corvées seigneuriales et surtout lorsque l'Etat fit construire gratuitement les fortifications de Québec et de Montréal. Si on

${ }^{6}$ La première menace de ce genre est du 10 juin 1732 (GV, 5: 42v.). On la trouvera à la fin de plusieurs autres procès-verbaux postérieurs.

7 A ce sujet, on pourra lire de Fernand Ouellet, La mentalité et l'outillage économique de l'habitant canadien (1760), dans BRH, (1956), vol. 62, no 3, pages 131-139.

8 Edits. ord., II : 366. 
n'aimait pas les corvées, c'était malgré tout le seul système connu des habitants.

Il y avait aussi d'autres motifs plus spécifiques à la voirie. En tête de tous les procès-verbaux, le grand-voyer affirmera que toutes les décisions ont été prises « après avoir Entendu Les dits habitants sus nommez [il s'agit des six qui l'ont accompagné, comme le voulait le grand règlement de voirie, et dont les noms se trouvaient toujours en tête des procès-verbaux] En leurs dires Et demandes touchant le dt Chemain ${ }^{9}{ }^{9}$ On prépare parfois la venue du grand-voyer en marquant les endroits les plus aptes à recevoir le chemin. ${ }^{10}$ Lorsqu'un procès-verbal est fait tôt le printemps, on fixe le début des travaux après les semences. ${ }^{11}$ On ordonne "qu'il sera travaillé sitost après Les Recoltes de la présente années pour Les terres Ensemmancées et que pour Celles qui sont En friche, Guéret ou Bois deBout il sera Incessamment travaillié $\gg .{ }^{12}$ Autant que possible, le tracé des chemins comme le temps des travaux correspondaient aux désirs des habitants. Sans doute, l'État obtenait-il ainsi plus facilement les corvées.

Mais l'explication la plus importante semble être la suivante: dès les 1710, les habitants ont eux-mêmes grandement besoin des chemins. On déclare souvent au grand-voyer avoir été «En soufrance d'un Chemin depuis Longtemps $\gg{ }^{13}$ En 1713, l'intendant Bégon écrira des habitants du Cap-Rouge que «n'ayant pu y travailler, a cause des travaux qui ont été faits l'année dernière pour les fortifications de cette ville [Québec], et comme ce même empêchement ne subsiste pas présentement, ils désireroient faire le dit chemin qui leur est absolument nécessaire $\gg .^{14}$ Ce texte est bien significatif, car ceux qui veulent faire le chemin ont déjà été chargés de corvées. Souvent les habitants feront venir euxmêmes le grand-voyer. ${ }^{15}$ Parfois, ils ont marqué et construit leur

${ }^{9} \mathrm{GV}, 1: 73 \mathrm{r}$. en 1707 et GV, $5: 2 \mathrm{v}$. en 1731.

$10 \mathrm{GV}, 5: 44 \mathrm{v}$.

$11 \mathrm{GV}, 1: 8 \mathrm{v}$.

$12 \mathrm{GV}, 5$ : 3r.

13 Ibid., 34 r.

14 Edits. ord., III : 433.

15 Nous avons relevé de tels exemples dans 18 seigneuries différentes. Elles se situent presque sans solution de continuité de Lanoraie à Montréal, de Portneuf à Québec et dans la banlieue des Trois-Rivières. Les seigneuries de Batiscan et de Champlain sont, en dehors du champs d'influence des villes, les seules où on trouve de telles requêtes. 
chemin sans attendre le grand-voyer. ${ }^{16} \mathrm{Si}$ on se passe parfois de6 secours spirituels ${ }^{17}$ ou médicaux ${ }^{18}$ faute de chemin, la plupart du temps les gens parlaient de «La Nécessite D'avoir un Chemin pour vacquer Librement a Leur affaire $\gg .^{19}$ Cette raison purement économique apparaît toujours la première dans les documents. En 1731, le gouverneur Beauharnois et l'intendant Hocquart écrivaient aux autorités de la Métropole que les habitants * ont par le moyen des chevaux des facilités pour transporter promptement dans les villes ou chez eux les bois à brûler, a bâtir, les matériaux et denrés dont ils ont besoin. ${ }^{20}$ Nous ne pouvons pas en douter: les habitants avaient eux-mêmes grandement besoin des chemins.

Enfin, une dernière raison! Dès 1706 , l'État a prévu contre les réfractaires des amendes. ${ }^{21}$ Leurs taux ont beaucoup varié: entre 3 et 20 livres. $^{22} \mathrm{Au}$ besoin, on fera remplacer les réfractaires à leur frais..$^{23}$ Le capitaine de milice tient un «Estat des Journées » des remplaçants, qui, remis à l'intendant, sera réglé «au dépend et Revenus dicelles [les terres] dont Les propriétaires ne se trouveront pas ou refuseront de travaillier $\gg,{ }^{24}$ et cela «outre et par dessus l'amende qu'ils auront Encouru par leurs désobéissance $»^{25}$ En ne se montrant presque jamais,

$16 \mathrm{GV}, 5: 41 \mathrm{r}$. et suiv.

$17 \mathrm{GV}, 1: 35 \mathrm{v}$.

$18 \mathrm{GV}, 5: 97 \mathrm{v}$.

19 Ibid., 26r., 97v.

20 Archives de la Province de Québec, Manuscrits relatifs à l'histoire de la Nouvelle-France, $3^{\mathrm{e}}$ série, vol. 12, lettre du 8, 8bre 1731 .

21 Elles vont au profit des fabriques de paroisses et le marguillier aura un droit de poursuite devant le juge de la seigneurie (OI, 17: 116v.). Nous connaissons trois exceptions à cette pratique: les amendes furent perçues au profit du capitaine de milice ( $E$ dits, ord., III: 418), à la réparation du chemin (Edits, ord., III : 433) et au soulagement des \& Pauvres, veuves des paroisses ou autres $\gg$ (Edits, ord., II : 341s.).

22 En général, lorsqu'il s'agit d'un premier procès-verbal et que les habitants sont bien disposés, les taux semblent moins élevés que lorsqu'on met difficilement fin à une longue contestation. Enfin, les amendes du grand-voyer seraient plutôt fixes, alors que l'intendant, plus libre devant les habitants et souvent amené à juger des cas plus difficiles, en adaptera les taux aux circonstances.

$23 \mathrm{GV}, 5$ : 53v.

24 Ibid., 27v., OI, 19: 130v.

25 OI, $6: 377 \mathrm{v}$. 
Bécancour n'avait pas pu rendre efficace ce système de punitions. Boiscler en tira de grandes réalisations.

En effet, avec 1735, toutes les seigneuries auront reçu le tracé de leur chemin. Dans les deux années qui suivent, Boiscler s'occupera principalement des bacs et des ponts. ${ }^{26}$ En 1737, seuls, quelques ponts ne seront pas faits. ${ }^{27} \mathrm{Si}$ nous considérons l'état lamentable des chemins et des ponts à l'arrivée de Boiscler en 1731 , il n'est sans doute pas faux de dire que le système des corvées a permis la construction du premier chemin royal de Québec à Montréal en 7 ans environ. Au cours de l'été 1737, il fut entièrement terminé et, l'année suivante, toute l'activité du grandvoyer pourra se porter ailleurs.

\section{CONCLUSION}

Le chemin allait maintenant rivaliser avec le fleuve. Les rives du lac Saint-Pierre, inondées tous les printemps, n'avaient pas été concédées: il aurait fallu construire les bâtiments trop loin à l'intérieur des terres et s'éloigner de la seule voie de communication, le fleuve. Hocquart écrira que le «nouveau chemin qui est sur une hauteur dans les terres et à couvert des inondations, a déjà engagé plusieurs habitants à prendre des concessions de ce côté $\gg .{ }^{28}$ Le chemin contribuait aux progrès de la colonie. Par ses courbes et ses côtes, ses trous et ses buttes, ses ponts parfois endommagés et la lenteur de ses bacs, il était sans doute fatigant pour les voyageurs. Mais avec ses 24 pieds de large, ses fossés, ses saignées-pontées et ses levées, il n'en restait pas moins très «praticable » et adapté aux besoins de l'époque. En 4 ou 6 jours, on pouvait aller de Québec à Montréal. ${ }^{29}$

Et ce premier chemin a été obtenu grâce aux corvées. Que pouvons-nous dire de ce système ? Un impôt (car la corvée est un impôt-travail), presqu'entièrement basé sur la propriété fon-

26 P.-G. Roy. Inventaire des procès-verbaux des grands-voyers, 90-96. 27 Ibid., $100 \mathrm{~s}$.

28 Lettre de Hocquart du 10 octobre 1732, citée par P.-G. Roy dans Les grands-voyers de la Nouvelle-France et leurs successeurs, Les Cahiers des Dix, No 8, 204.

29 Franquet, Voyages ..., 168. 
cière, convenait-il à la situation du Canada de l'époque ? N'étaitil pas brutal d'exiger d'un habitant qui n'a pas défriché sa concession le même travail que celui qui en avait des revenus satisfaisants ? Ceci s'explique par la pensée des autorités qui voient dans le concessionnaire-coureur de bois un paysan négligent. Si plusieurs ne tirent aucun profit de leur terre, l'État les en tient responsables et n'a par conséquent aucune faveur à leur faire.

De plus, tout nous porte à croire que le roi s'est peu intéressé à la construction du chemin. Ne continue-t-il pas à payer un Bécancour vieillissant et invalide ? ${ }^{30}$ Il prend du temps à lui nommer un successeur. ${ }^{31}$ En 1734, Hocquart lui annonce vaguement que la «colonie en [du chemin] retirera bientôt tous les avantages que l'on s'étoit proposé depuis un grand nombre d'années d'en retirer $\gg^{32}$

L'entreprise aurait donc son origine dans la colonie. Elle entre bien d'ailleurs dans le grand programme de développement économique de Hocquart. Mais d'autre part, la plupart des seigneurs de la rive nord du fleuve se retrouvent parmi les grands commerçants des villes ou les fonctionnaires de l'État. Ils ont probablement le plus besoin des chemins pour leurs affaires commerciales ou administratives. On peut se demander si ce n'est pas sous leur pression plus ou moins directe qu'on a forcé tous les gens à construire le chemin. On expliquerait ainsi pourquoi les seigneurs, qui d'ailleurs n'avaient pas l'ancienneté et le prestige des seigneurs de France, ne furent pas dispensés des corvées et pourquoi plusieurs habitants refusaient de faire leur part des travaux.

Le régime de la corvée lui-même peut être mis en cause. Songeons à l'importance de l'argent en Nouvelle-France, aux besoins de main-d'œuvre, aux bons salaires offerts dans la construction navale. Lorsqu'on remplace un contrevenant, le prix d'une journée de travail d'un paysan sera remboursé par le con$8,199$.

$30 \mathrm{Il}$ mourut en 1729 à l'âge de 75 ans. Voir Les Cahiers des Dix, no

$31 \mathrm{APQ}$. Manuscrits relatifs à l'histoire de la Nouvelle-France, $3^{\mathrm{e}}$ série, vol. 11, 2352.

32 Edits, ord., II : 366. 
trevenant «sauf qu'il puisse passer quarante sols [deux livres], ainsi que la journée d'un charpentier, s'il y était nécessaire, plus de trois livres $\gg .^{33} \mathrm{Si}$ on connaissait toujours le principe des corvées, ces faits prouvent qu'on avait aussi l'habitude de penser au travail en raison du salaire immédiat et qu'on reconnaissait le travail spécialisé. Telle n'est pas la situation du corvéable et le charpentier soumis à la corvée fournit plus de travail (en valeur) que le paysan. Ce conflit entre le régime du salariat et celui de la corvée existait-il suffisamment pour être une autre cause de difficultés ?...

C'est là une autre question à laquelle une histoire plus poussée de l'économie canadienne sous le régime français pourrait répondre. Alors seulement on pourrait voir la position exacte occupée par le système de la corvée dans la société canadienne d'avant la Conquête.

RolaNd SANFaÇon, L. ès L. (histoire)

33 Edits, ord., II : 341.

\section{PRIĖRE À NOS ABONNÉS:}

Vous aimez la Revue? Faites-la connaître dans votre milieu. Et obtenez-nous de nouveaux abonnés.

L. G. 